\title{
Functional mobility trajectories of hospitalized older adults admitted to acute geriatric wards: A retrospective observational study in an English university hospital
}

\begin{abstract}
Adam Lyons, ${ }^{1,2}$ Roman Romero-Ortuno ${ }^{2,3,4}$ (ID and Peter Hartley ${ }^{5,6}$ (D)
${ }^{1}$ School of Clinical Medicine,

University of Cambridge,

Cambridge, UK

${ }^{2}$ Department of Medicine for the

Elderly, Addenbrooke's Hospital,

Cambridge, UK

${ }^{3}$ Clinical Gerontology Unit,

Department of Public Health and

Primary Care, University of

Cambridge, Cambridge, UK

${ }^{4}$ Discipline of Medical

Gerontology, Trinity College
\end{abstract}

Dublin, Mercer's Institute for

Successful Aging, St James's

Hospital, Dublin, Ireland

${ }^{5}$ Primary Care Unit, Department of

Public Health and Primary Care,

University of Cambridge,

Cambridge, UK

${ }^{6}$ Department of Physiotherapy,

Addenbrooke's Hospital,

Cambridge, UK

\section{Correspondence}

Mr Peter Hartley MSc, Cambridge Institute of Public Health, Forvie Site, University of Cambridge School of Clinical Medicine, Cambridge Biomedical Campus, Cambridge CB2 OSR, UK.

Email: ph492@medschl.cam.ac.uk

Received: 21 September 2018

Revised: 19 November 2018

Accepted: 1 January 2019

\section{Introduction}

Many older people experience a loss of function in response to a stressor, such as an illness or fall. ${ }^{1}$ As a consequence, many are admitted to hospital having experienced a loss of function associated with their acute illness. ${ }^{2,3}$ In those cases, an objective of hospital care is to rehabilitate that loss of function, and minimize the degree of illness-associated functional loss. As a result, samples of hospitalized patients show an average improvement in functional mobility scores from hospital admission to hospital discharge. ${ }^{4-6}$

In summarizing the change in functional mobility in a sample of hospitalized patients as the average change from admission to discharge, we risk oversimplification and misunderstanding. For instance, this improvement in function might not represent complete resolution to a pre-illness level, ${ }^{7,8}$ and further, a small but significant number of patients are known to deteriorate in function from admission to discharge. ${ }^{2,9,10}$ This indicates that there might be distinctive subsets of patients with different functional trajectories. A greater understanding of the patient characteristics within the different subsets might improve our understanding of patients at risk of poor hospital outcome, and in time lead to improved hospital care. The aim of the present study therefore was to identify distinct subsets of patients based on similar functional mobility trajectories, and to describe the patient characteristics and outcomes of the different subsets.

\section{Methods}

\section{Design and setting}

We carried out a retrospective observational study of all first-time admissions to the Department of Medicine for the Elderly (DME) 
wards of Addenbrooke's Hospital, which is a tertiary university National Health Service acute hospital in England, for 3 months starting 1 May 2016. The setting has been described elsewhere. ${ }^{11,12}$ In brief, the six DME wards specialize in ward-based Comprehensive Geriatric Assessment. All patients who are admitted to DME wards are routinely assessed by a physiotherapist, and the dedicated nursing and medical teams are able to refer to other allied health professionals (e.g. occupational therapy, dietetics, speech and language therapy, social work) or other medical specialties or psychiatry for old age if their inputs are required.

\section{Measures}

Anonymized routinely collected clinical data were extracted from the electronic patient records. Information extracted included:

- Age (years) and sex.

- Clinical Frailty Scale (CFS). A local Commissioning for Quality and Innovation scheme introduced in 2013 made it a mandatory requirement that all patients aged $>75$ years were to be screened for frailty using the CFS within $72 \mathrm{~h}$ of admission if they were admitted through the emergency pathway. A section with the CFS and its scoring instructions (as per http:// geriatricresearch.medicine.dal.ca/clinical_frailty_scale.htm) was included in the standard electronic medical admission pro forma. The admitting team usually scored the CFS on the pro forma, as described elsewhere. ${ }^{11}$

- A diagnosis of dementia, based on a review of the medical notes.

- A diagnosis of delirium, based on a review of the medical notes.

- Charlson Comorbidity Index.

- The number of falls in the period of approximately 1 year before hospital admission (as recalled by the patient or a proxy and recorded in the hospital chart during routine historytaking).

- Acute illness severity on admission, measured by the highest recorded Modified Early Warning Score in the Emergency Department. ${ }^{13}$ Early warning scores are routinely used in National Health Service hospitals.

- The Elderly Mobility Scale (EMS), a scale of functional mobility from $0-20$, with 0 representing high dependence. ${ }^{14}$ Physiotherapists routinely collected the EMS on admission and discharge. The scale includes the assessment of balance, mobility and ability to change body positions (e.g. from lying to sitting). The interrater reliability of the EMS has been reported as $r=0.88$ $(P<0.001)$, and it has good convergent validity with the Barthel Index $(r=0.787, P<0.001) .{ }^{15}$ The minimally clinically important difference of the EMS has been estimated at 2 points. ${ }^{16}$

- Total length of stay (days).

- The place of residence before admission.

- The discharge destination.

\section{Statistical analysis}

Anonymized data were analyzed with IBM sPSs Statistics (version 22; IBM, Armonk, NY, USA) software, and images produced with $\mathrm{R}$ software, ${ }^{17}$ using the ggplot2 package. ${ }^{18}$ Descriptive statistics were given as the number (with percentage) or median (interquartile range). When comparing characteristics of those patients who died during the hospital admission with those survived, the Wilcoxon rank sum test with continuity correction was used for continuous variables, and Pearson's $\chi^{2}$-test with Yates' continuity correction for categorical variables. The two EMS variables (admission and discharge) were entered in a K-means cluster analysis to produce five different mobility trajectory groups. Multivariate binary logistic regression models were carried out to look for independent predictors of membership of each group. In those models, the variables used as independent predictors were: age, sex, Modified Early Warning Score in the Emergency Department, frailty, delirium, dementia and the number of falls in the past 12 months. Inpatient deaths were excluded from the present study, as well as those without EMS data on either admission or discharge.

\section{Ethics approval}

This study was approved as a service evaluation by our center's Safety and Quality Support Department (Project Register Number 5205).

\section{Results}

Of the 926 first-time admissions to the DME wards during the 3 months, 856 (92\%) survived from admission to discharge. Patients who died during admission had a higher CFS score $(P<0.001)$, Modified Early Warning Score in the Emergency

Table 1 Characteristics of each mobility trajectory group, as well as that of the whole cohort

\begin{tabular}{|c|c|c|c|c|c|c|}
\hline Variable & Total $(n=715)$ & LL $(n=117)$ & II $(n=95)$ & $\mathrm{IH}(n=205)$ & LH $(n=89)$ & $\mathrm{HH}(n=209)$ \\
\hline Female & $413(58 \%)$ & $75(64 \%)$ & $54(57 \%)$ & $120(59 \%)$ & $53(60 \%)$ & $111(53 \%)$ \\
\hline Age (years) & $86(81-90)$ & 87 (80-91) & $86(81-91)$ & $87(83-92)$ & $86(81-90)$ & $84(79-88)$ \\
\hline CFS & $6(5-6)$ & $7(6-7)$ & $6(6-7)$ & $6(5-6)$ & $6(5-6)$ & $4(3-5)$ \\
\hline Highest ED-MEWS & $2(2-4)$ & $3(2-4)$ & $2(2-4)$ & $2(1-3)$ & $2(2-4)$ & $2(1-4)$ \\
\hline EMS at admission & $11(5-16)$ & $0(0-2)$ & $8(6-10)$ & $12(11-14)$ & $3(0-6)$ & $18(16-20)$ \\
\hline EMS at discharge & $14(9-18)$ & $0(0-3)$ & $8(6-10)$ & $14(12-15)$ & $15(13-17)$ & $18(17-20)$ \\
\hline EMS decreased by $\geq 2$ during hospitalization & $65(9 \%)$ & $15(13 \%)$ & $25(26 \%)$ & $15(7 \%)$ & $0(0 \%)$ & $10(5 \%)$ \\
\hline EMS improved by $\geq 2$ during hospitalization & $267(38 \%)$ & $23(20 \%)$ & $25(26 \%)$ & $90(44 \%)$ & $89(100 \%)$ & $40(19 \%)$ \\
\hline Falls in last 12 months & $1(0-3)$ & $0(0-3)$ & $1.5(0-5)$ & $2(0-3)$ & $1.5(0-4)$ & $1(0-2)$ \\
\hline Delirium & $135(19 \%)$ & $37(32 \%)$ & $23(24 \%)$ & $33(16 \%)$ & $22(25 \%)$ & $20(10 \%)$ \\
\hline Dementia & $199(28 \%)$ & $53(45 \%)$ & $44(46 \%)$ & $52(25 \%)$ & $22(24 \%)$ & $28(13 \%)$ \\
\hline CCI & $2(1-3)$ & $2(1-3)$ & $2(1-3)$ & $2(1-3)$ & $1(0-3)$ & $1(1-3)$ \\
\hline Length of stay (days) & $7(3-15)$ & $11(6-20)$ & $7(3-17)$ & $6(3-13)$ & $15(8-27)$ & $4(2-7)$ \\
\hline \multicolumn{7}{|l|}{ Normal residence } \\
\hline Own home & $606(85 \%)$ & $72(62 \%)$ & $72(76 \%)$ & $180(88 \%)$ & $81(91 \%)$ & $201(96) \%$ \\
\hline Residential home & $76(11 \%)$ & $25(21 \%)$ & $19(20 \%)$ & $21(10 \%)$ & $6(7 \%)$ & $5(2 \%)$ \\
\hline Nursing home & $32(4 \%)$ & $20(17 \%)$ & $4(4 \%)$ & $4(2 \%)$ & $2(2 \%)$ & $2(1 \%)$ \\
\hline New institutionalization & $100(14 \%)$ & $32(27 \%)$ & $22(23 \%)$ & $24(12 \%)$ & $15(16 \%)$ & $8(4 \%)$ \\
\hline
\end{tabular}

Values are presented as the number (percentage) or as the median (interquartile range). CCI, Charlson Comorbidity Index; CFS, Clinical Frailty Scale; ED-MEWS, Modified Early Warning Score in the Emergency Department; EMS, Elderly Mobility Scale; HH, high-high. II, intermediate-intermediate; IH, intermediate-high; LH, low-high; LL, low-low. 


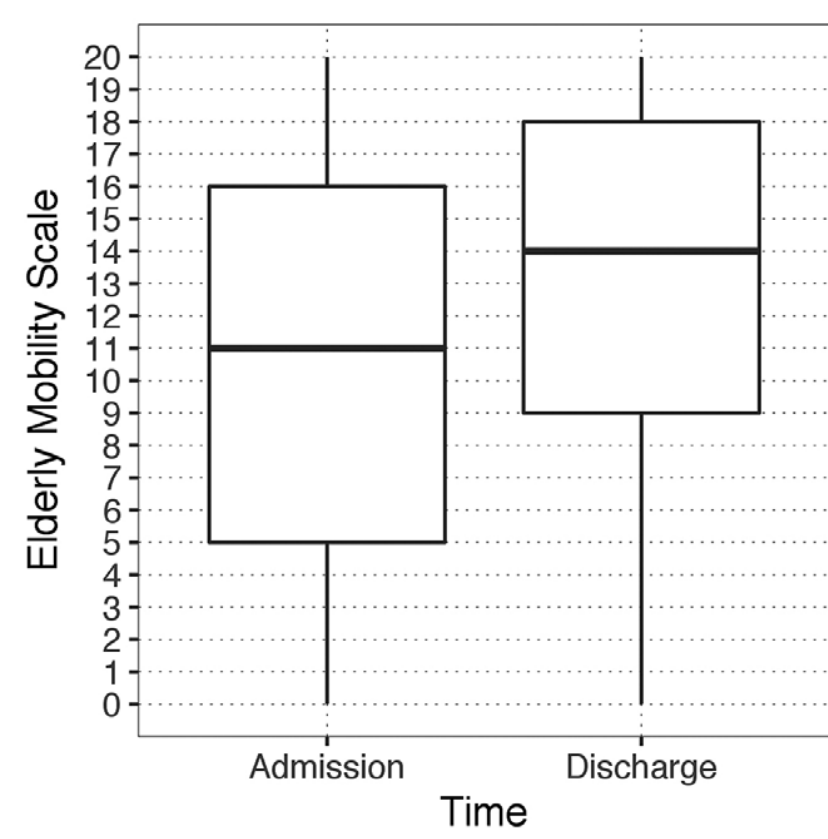

Figure 1 Elderly Mobility Scale on admission and discharge for the whole cohort $(n=715)$.

Department $(P<0.001)$ and Charlson Comorbidity Index $(P=0.001)$ compared with those who survived (see Supporting Information Table S1). Of the patients who survived, 715 (77\% of first time admissions) had EMS scores on admission and discharge. The median age of the cohort was 86 years ( $81-90$ years), and $58 \%$ were women (Table 1 ).

The functional trajectory from admission to discharge for the entire sample $(n=715)$ is presented in Figure 1. The median EMS score increased from $11(5-16)$ on admission to 14 (9-18) on discharge. A total of $65(9 \%)$ of the patients experienced a loss on the EMS of $\geq 2$ points, whereas 267 (38\%) had an improvement of $\geq 2$ points.

The K-means cluster analysis identified five different mobility trajectory groups. These mobility groups were named based on their median admission and discharge EMS scores: low-low (LL, $n=117$ ), intermediate-intermediate (II, $n=95$ ), intermediate-high
(IH, $n=205)$, low-high (LH, n=89) and high-high (HH, $n=209$ ). Figure 2 shows a graphical representation of the mobility trajectories of these subgroups, and Table 1 shows their characteristics, as well as those of the overall cohort.

Multivariate logistic regression analyses (Table 2) suggested that those in the LL and II groups had a higher burden of both frailty and cognitive impairment. Frailty was also a predictor of membership to the LH group, but those with a history of dementia seemed to be less represented in this group compared with the LL and II groups. Membership to the IH group seemed to be predicted by higher age and lower acute illness severity. Lower age, lower frailty and absence of delirium significantly increased the odds of membership to the HH group.

\section{Discussion}

The present study retrospectively identified five distinct subsets of patients based on similar functional mobility trajectories, and identified key patient characteristics associated with each subset. The benefit of this methodology is in highlighting the heterogeneity of functional trajectories observed in geriatric wards. Although we observed an overall improvement in functional mobility of more than the reported minimally clinically important difference of 2 points on the EMS, this threshold of 2 points was only observed in two of the five groups ( $\mathrm{LH}$ and $\mathrm{IH}$ ). Frailty and cognitive impairment in combination were associated with membership of groups with poor functional mobility at discharge from hospital (II and LL).

The overall functional trajectory across this cohort from a median EMS score of 11 on admission to 14 on discharge is a key service evaluation finding. Our local finding could provide some comfort and confidence to patients and clinicians, and help allay common popular fears that a hospitalization tends to carry a high risk of negative functional outcome in all older adults. ${ }^{19}$ However, this does obscure some of the underlying heterogeneity. We observed that for some people, there is a significant loss of function ( $9 \%$ had a drop in EMS score of $\geq 2$ points). This shows that while in general there is improvement and on average there appears to be a positive impact, subgroup analyses are key to the better understanding of patient outcomes. ${ }^{20}$

In our K-means cluster analysis, we pre-determined five clusters in order to illustrate the underlying heterogeneity of the
Figure 2 Median Elderly Mobility Scale on admission and discharge for five groups after Kmeans cluster analysis. Shaded areas represent the interquartile ranges.

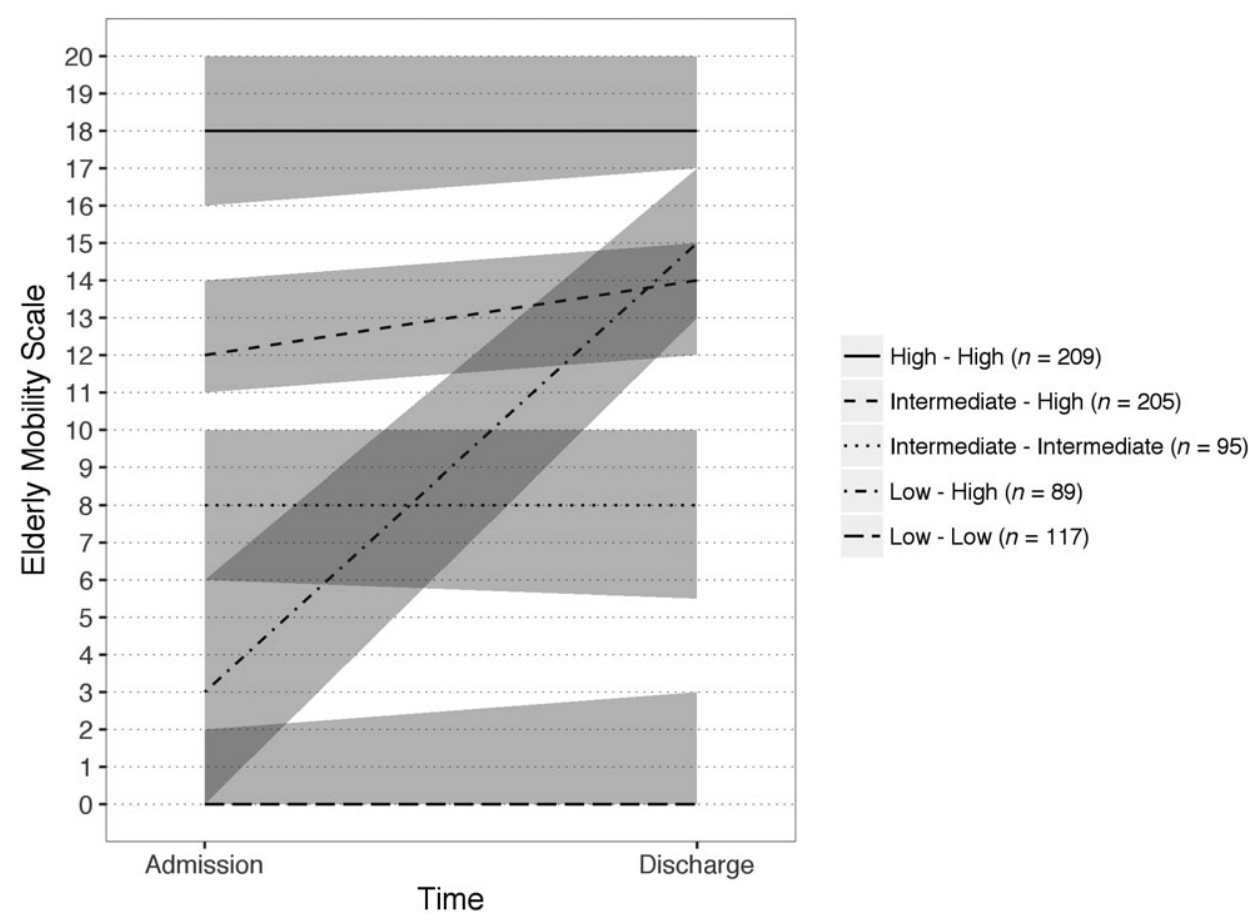


sample, but we do not imply that five is necessarily the most statistically efficient number of clusters. However, the characteristics of the clusters might resonate in clinicians' minds as patterns that are commonly seen in real clinical practice. At one end, we identified those that tend to be admitted with a high level of mobility, from their own home and with low levels of cognitive impairment; they have comparatively low levels of frailty, and are discharged to their own home having maintained their mobility and function, thus having a low percentage of new institutionalization (HH group). At the other end, we identified those who tend to be severely frail when admitted, more often from residential and nursing homes, and with low baseline mobility scores; they have comparatively high levels of cognitive impairment, do not significantly improve their mobility during hospitalization and are at risk of new institutionalization (LL group). Some admissions in the LL group might be from the community when care for an advanced chronic disabling process can no longer be provided in the community. In between these groups, those in the LH group tend to experience a marked improvement in mobility while in hospital, perhaps as a result of an acute illness that limits mobility acutely, but gradually wears off over a longer period of hospitalization.
There are also the groups that start off at intermediate mobility and then diverge, the II and IH groups. The LL, II and LH groups are of most interest, with the prior two being the more negative functional patterns and the latter being the most positive pattern in terms of the effect of hospitalization on mobility. Research is required to understand how best to optimize functional outcomes for high complexity patients, and that involves moving patients from the LL and II groups into LH groups. However, that was not in the scope of the present study.

The present results suggest a potential relationship between clinical frailty and a poor level of functional mobility on admission. This is in part explained by the scoring of the CFS including disability (as well as symptoms, comorbidity, cognition and the overall clinician's impression). It is therefore logical that there is a degree of overlap between our definition of frailty and disability (functional mobility). The logistic regression models show increased odds of membership to the LL, II and LH group with increased frailty. This is in keeping with the findings of our previous work. ${ }^{5}$ Other work has shown increased frailty to be associated with increased odds of a negative functional outcome, as in the LL and II group. ${ }^{21}$ However, it is interesting to note that the

Table 2 Multivariable predictors of outcomes

\begin{tabular}{|c|c|c|c|c|c|}
\hline & & \multirow[t]{2}{*}{ Odds ratio (OR) } & \multicolumn{2}{|c|}{$95 \% \mathrm{CI}$ for OR } & \multirow[t]{2}{*}{$P$} \\
\hline & & & Lower & Upper & \\
\hline \multirow[t]{8}{*}{ Low-low $(n=117)$} & Sex & 1.124 & 0.686 & 1.841 & 0.643 \\
\hline & Age & 0.997 & 0.958 & 1.037 & 0.881 \\
\hline & Frailty & 2.311 & 1.788 & 2.987 & $<0.001 *$ \\
\hline & ED-MEWS & 1.101 & 0.949 & 1.276 & 0.204 \\
\hline & Falls & 1.000 & 0.976 & 1.024 & 0.992 \\
\hline & Delirium & 1.989 & 1.197 & 3.306 & $0.008 *$ \\
\hline & Dementia & 1.308 & 0.795 & 2.150 & 0.290 \\
\hline & $\mathrm{CCI}$ & 1.017 & 0.890 & 1.162 & 0.804 \\
\hline \multirow[t]{8}{*}{ Intermediate-intermediate $(n=95)$} & Sex & 0.845 & 0.517 & 1.379 & 0.500 \\
\hline & Age & 0.977 & 0.938 & 1.018 & 0.271 \\
\hline & Frailty & 1.544 & 1.238 & 1.926 & $<0.001 *$ \\
\hline & ED-MEWS & 0.980 & 0.840 & 1.142 & 0.793 \\
\hline & Falls & 1.022 & 0.993 & 1.051 & 0.135 \\
\hline & Delirium & 1.069 & 0.608 & 1.877 & 0.817 \\
\hline & Dementia & 1.730 & 1.032 & 2.899 & $0.038 *$ \\
\hline & CCI & 1.068 & 0.936 & 1.218 & 0.326 \\
\hline \multirow[t]{8}{*}{ Intermediate-high $(n=205)$} & Sex & 1.057 & 0.735 & 1.520 & 0.765 \\
\hline & Age & 1.059 & 1.027 & 1.093 & $<0.001 *$ \\
\hline & Frailty & 1.016 & 0.884 & 1.168 & 0.825 \\
\hline & ED-MEWS & 0.873 & 0.774 & 0.985 & $0.027 *$ \\
\hline & Falls & 0.997 & 0.974 & 1.021 & 0.828 \\
\hline & Delirium & 0.750 & 0.471 & 1.192 & 0.223 \\
\hline & Dementia & 0.745 & 0.480 & 1.157 & 0.190 \\
\hline & $\mathrm{CCI}$ & 1.092 & 0.988 & 1.207 & 0.085 \\
\hline \multirow[t]{8}{*}{ Low-high $(n=89)$} & Sex & 1.021 & 0.622 & 1.676 & 0.935 \\
\hline & Age & 0.991 & 0.952 & 1.032 & 0.671 \\
\hline & Frailty & 1.325 & 1.090 & 1.610 & $0.005^{*}$ \\
\hline & ED-MEWS & 1.000 & 0.858 & 1.166 & 0.998 \\
\hline & Falls & 0.982 & 0.929 & 1.038 & 0.523 \\
\hline & Delirium & 1.296 & 0.732 & 2.292 & 0.374 \\
\hline & Dementia & 0.541 & 0.295 & 0.994 & $0.048 *$ \\
\hline & CCI & 0.936 & 0.813 & 1.077 & 0.355 \\
\hline \multirow[t]{8}{*}{ High-high $(n=209)$} & Sex & 0.882 & 0.578 & 1.347 & 0.562 \\
\hline & Age & 0.959 & 0.924 & 0.996 & $0.028 *$ \\
\hline & Frailty & 0.453 & 0.381 & 0.539 & $<0.001 *$ \\
\hline & ED-MEWS & 1.039 & 0.908 & 1.189 & 0.580 \\
\hline & Falls & 0.930 & 0.859 & 1.008 & 0.076 \\
\hline & Delirium & 0.469 & 0.250 & 0.882 & $0.019 *$ \\
\hline & Dementia & 0.967 & 0.547 & 1.712 & 0.910 \\
\hline & CCI & 0.900 & 0.796 & 1.016 & 0.088 \\
\hline
\end{tabular}

*Statistically significant results $(P<0.05)$. CCI, Charlson Comorbidity Index; CI, confidence interval; ED-MEWS, Modified Early Warning Score in the Emergency Department; OR, odds ratio. 
LH group shows a vast improvement in their EMS scores despite membership of the group being associated with frailty. This suggests that even though some patients might be very frail, they still have significant potential for improvement in their function during hospitalization. This might suggest that frailty per se is not necessarily associated with adverse functional patterns, instead, it appears that frailty in combination with other geriatric syndromes, such as cognitive impairment, is associated with negative mobility trajectories.

Cognitive impairment has previously been shown to be associated with poor functional patterns. ${ }^{22}$ In our sample, the prevalence of dementia and delirium are broadly in line with those reported in other hospitals. ${ }^{23,24}$ The LL and II subgroups had the lowest EMS scores at discharge, and the highest percentage of patients with a clinically significant deterioration in the EMS scores, and were the only groups in which the presence of cognitive impairment was associated with increased odds of membership (Table 2). Despite comparative levels of delirium and frailty in the II and LH groups ( $24.2 \%$ and $24.7 \%$ ), the groups had very different functional trajectories. This might be explained by the higher prevalence of dementia in the II group, or potential differences in the severity and duration of delirium, which we did not capture in the data. Delirium superimposed on dementia has been hypothesized to be a strong predictor of poor functional performance, and has been shown to be so in studies of patients undergoing rehabilitation. ${ }^{25,26}$ It is difficult to disentangle these different possibilities using our current analysis, which is one of the various limitations of the present study.

A key service evaluation finding is that $77 \%$ of the eligible sample had EMS scores on admission and discharge, which underscores the "real-life" nature of the acute hospital setting in which the study was carried out. Those who did not have complete EMS data included those who died during the inpatient stay, and this highlights the known association between missing information in performance-based measures and adverse outcomes in clinical databases. ${ }^{27}$

This was a retrospective study with a single center focus and thus the results are not generalizable or externally valid. In addition, although associations can be identified between the predictors used in the logistic regression models and the mobility patterns, causal links cannot be inferred. In the present study, baseline function before hospitalization was not recorded, so we do not know how close to their baseline patients were when they were discharged and thus how "well" they recovered. We did not collect the admission diagnosis, which could act as a potential confounding variable for our cluster analysis. Furthermore, we have relied on routinely collected clinical data, variables such as the diagnosis of dementia or delirium might therefore underestimate the true prevalence. ${ }^{24,28}$

The present study underscores the importance of the routine and early identification of geriatric syndromes in the acute hospital, and how they are key to understanding the outcomes of a naturally heterogeneous population. Frailty alone was not associated with poor functional outcome, and should not be considered a barrier to functional improvement. Research is necessary to establish whether interventions outside "usual care," such as enhanced physical and/or cognitive therapy, could improve some of the adverse functional trajectories in the most vulnerable subgroups.

\section{Acknowledgements}

We thank all the members of the DME teams in our hospital, without whom this initiative would not have been possible.

\section{Disclosure statement}

The authors declare no conflict of interest.

\section{References}

1 Clegg A, Young J, Iliffe S, Rikkert MO, Rockwood K. Frailty in elderly people. Lancet 2013; 381: 752-762.

2 Mudge AM, O'Rourke P, Denaro CP. Timing and risk factors for functional changes associated with medical hospitalization in older patients. J Gerontol A Biol Sci Med Sci 2010; 65: 866-872.

3 Sleiman I, Rozzini R, Barbisoni P et al. Functional trajectories during hospitalization: a prognostic sign for elderly patients. J Gerontol A Biol Sci Med Sci 2009; 64: 659-663.

4 Bodilsen AC, Pedersen MM, Petersen J et al. Acute hospitalization of the older patient: changes in muscle strength and functional performance during hospitalization and 30 days after discharge. Am J Phys Med Rehabil 2013; 92: 789-796.

5 Hartley P, Adamson J, Cunningham C, Embleton G, RomeroOrtuno R. Clinical frailty and functional trajectories in hospitalized older adults: a retrospective observational study. Geriatr Gerontol Int 2017; 17: 1063-1068.

6 Palleschi L, De Alfieri W, Salani B et al. Functional recovery of elderly patients hospitalized in geriatric and general medicine units. The PROgetto DImissioni in GEriatria Study. J Am Geriatr Soc 2011; 59: 193-199.

7 Zisberg A, Shadmi E, Gur-Yaish N, Tonkikh O, Sinoff G. Hospitalassociated functional decline: the role of hospitalization processes beyond individual risk factors. J Am Geriatr Soc 2015; 63: 55-62.

8 Fimognari FL, Pierantozzi A, De Alfieri W et al. The severity of acute illness and functional trajectories in hospitalized older medical patients. J Gerontol A Biol Sci Med Sci 2017; 72: 102-108.

9 D'Onofrio A, Bula C, Rubli E, Butrogno F, Morin D. Functional trajectories of older patients admitted to an Acute Care Unit for Elders. Int $J$ Older People Nurs 2018; 13: e12164.

10 Pedone $\mathrm{C}$, Ercolani S, Catani $\mathrm{M}$ et al. Elderly patients with cognitive impairment have a high risk for functional decline during hospitalization: the GIFA Study. J Gerontol A Biol Sci Med Sci 2005; 60: 1576-1580.

11 Wallis SJ, Wall J, Biram RW, Romero-Ortuno R. Association of the clinical frailty scale with hospital outcomes. QJM 2015; 108: 943-949.

12 Romero-Ortuno R, Forsyth DR, Wilson KJ et al. The Association of geriatric syndromes with hospital outcomes. J Hosp Med 2017; 12: 83-89.

13 Romero-Ortuno R, Wallis S, Biram R, Keevil V. Clinical frailty adds to acute illness severity in predicting mortality in hospitalized older adults: an observational study. Eur J Intern Med 2016; 35: 24-34.

14 Smith R. Validation and reliability of the elderly mobility scale. Physiotherapy 1994; 80: 744-747.

15 Prosser L, Canby A. Further validation of the Elderly Mobility Scale for measurement of mobility of hospitalized elderly people. Clin Rehabil 1997; 11: 338-343.

16 de Morton NA, Berlowitz DJ, Keating JL. A systematic review of mobility instruments and their measurement properties for older acute medical patients. Health Qual Life Outcomes 2008; 6: 44.

17 R Core Team. R: A Language and Environment for Statistical Computing. Vienna, Austria.: R Foundation for Statistical Computing, 2018. Retrieved from URL: https://www.R-project.org/.

18 Wickham, H. ggplot2: Elegant Graphics for Data Analysis. New York: Springer-Verlag, 2016

19 Mail Online. How just 10 days in hospital can make a patient 10 years older. 2016. Available from URL: https://www.dailymail.co. uk/health/article-3609849/How-just-10-days-hospital-make-patient10-years-older.html. Accessed July 2018.

20 Mitnitski A, Howlett SE, Rockwood K. Heterogeneity of human aging and its assessment. J Gerontol A Biol Sci Med Sci 2017; 72: 877-884.

21 Hatheway OL, Mitnitski A, Rockwood K. Frailty affects the initial treatment response and time to recovery of mobility in acutely ill older adults admitted to hospital. Age Ageing 2017; 46: 920-925.

22 Hartley P, Gibbins N, Saunders A et al. The association between cognitive impairment and functional outcome in hospitalised older patients: a systematic review and meta-analysis. Age Ageing 2017; 46: 559-567.

23 Timmons S, Manning E, Barrett A et al. Dementia in older people admitted to hospital: a regional multi-hospital observational study of prevalence, associations and case recognition. Age Ageing 2015; 44: 993-999.

24 Ryan DJ, O'Regan NA, Caoimh RO et al. Delirium in an adult acute hospital population: predictors, prevalence and detection. BMJ Open 2013; 3: e001772.

25 Morandi A, Davis D, Fick DM et al. Delirium superimposed on dementia strongly predicts worse outcomes in older rehabilitation inpatients. J Am Med Dir Assoc 2014; 15: 349-354.

26 Fick DM, Steis MR, Waller JL, Inouye SK. Delirium superimposed on dementia is associated with prolonged length of stay and poor outcomes in hospitalized older adults. J Hosp Med 2013; 8: 500-505.

27 Rockwood K, Jones D, Wang Y, Carver D, Mitnitski A. Failure to complete performance-based measures is associated with poor health status and an increased risk of death. Age Ageing 2007; 36: 225-228.

28 Sommerlad A, Perera G, Singh-Manoux A, Lewis G, Stewart R, Livingston G. Accuracy of general hospital dementia diagnoses in England: sensitivity, specificity, and predictors of diagnostic accuracy 2008-2016. Alzheimers Dement 2018; 14: 933-943. 


\section{Supporting information}

Additional supporting information may be found in the online version of this article at the publisher's website.

Table S1 Admission characteristics of patients who survived admission and those who died during their admission. Values are presented as the number (percentage) or as the median (interquartile range).
How to cite this article: Lyons A, Romero-Ortuno R, Hartley P. Functional mobility trajectories of hospitalized older adults admitted to acute geriatric wards: A retrospective observational study in an English university hospital. Geriatr. Gerontol. Int. 2019;19:305-310. https://doi.org/10. 1111/ggi.13623 